\title{
Finite homomorphism-homogeneous permutations via edge colourings of chains
}

\author{
Igor Dolinka Éva Jungábel \\ Department of Mathematics and Informatics \\ Faculty of Science, University of Novi Sad \\ Trg Dositeja Obradovića 4, 21101 Novi Sad, Serbia \\ dockie@dmi.uns.ac.rs, eva.jungabel@dmi.uns.ac.rs \\ Submitted: Apr 20, 2012; Accepted: Oct 23, 2012; Published: Nov 1, 2012 \\ Mathematics Subject Classifications: 05A05, 03C07, 06A05, 06A06
}

\begin{abstract}
A relational structure is homomorphism-homogeneous if any homomorphism between its finite substructures extends to an endomorphism of the structure in question. In this note, we characterise all permutations on a finite set enjoying this property. To accomplish this, we switch from the more traditional view of a permutation as a set endowed with two linear orders to a different representation by a single linear order (considered as a directed graph with loops) whose non-loop edges are coloured in two colours, thereby 'splitting' the linear order into two posets.
\end{abstract}

Keywords: homomorphism-homogeneous, finite permutation, linear order

\section{Introduction}

First of all there is Blue. Later there is White, and then there is Black, and before the beginning there is Brown.

Paul Auster: Ghosts (The New York Trilogy)

$$
* \quad * \quad *
$$

A first-order structure $\mathcal{A}$ is called ultrahomogeneous (or simply homogeneous) if any partial automorphism of $\mathcal{A}$ defined on its finitely generated substructure is a restriction of some automorphism of $\mathcal{A}$. If we confine ourselves to relational structures (which is exactly what we do in this note), then this property amounts to saying that any isomorphism between finite (induced) substructures of $\mathcal{A}$ extends to an automorphism of $\mathcal{A}$. Homogeneity is a definite indicator of an extremely high level of symmetry present within a 
structure, in other words, of the 'richness' of its automorphism group. Together with the related notions of amalgamation, Fraïssé limits, quantifier elimination etc. [12, 14, 21], homogeneous structures constitute nowadays a classical chapter of model theory. For a multitude of particular classes of structures, homogeneous members have been fully classified; for example, this is the case with finite graphs [13], countably infinite graphs [19], countable tournaments [18], countable partially ordered sets [29], finite groups [9], countable directed graphs [8] and countable permutations [5].

In their seminal paper [7], Cameron and Nešetřil examine variations of the notion of homogeneity that involve conditions of extending different types of homomorphism of a structure. For instance, a relational structure $\mathcal{A}$ is said to be homomorphismhomogeneous if any homomorphism $\mathcal{B} \rightarrow \mathcal{B}^{\prime}$ between finite substructures of $\mathcal{A}$ extends to an endomorphism of $\mathcal{A}$. As amply showed in [26], it is precisely this property that captures a similar kind of 'richness' and symmetry in the endomorphism monoid of a structure as (ultra)homogeneity does in its automorphism group. A classification theory of homomorphism-homogeneous structures seems to be emerging quickly since then (see e.g. $[6,11,15,16,22,23,25])$, while such structures also harbour some classes of high computational complexity $[24,28]$, which therefore may be considered 'unclassifiable'. Incidentally, homomorphism-homogeneous groups trace back to $[3,30]$ under the guise of a similar (and in the finite case, equivalent) property of quasi-injectivity.

It is the goal of this note to provide a full description of all finite permutations enjoying the property of homomorphism-homogeneity. Following, for example, the enlightening exposition of [5], recall that a permutation is a relational structure $\pi=\left(A,<_{1},<_{2}\right)$, where the underlying set $A$ is equipped with two (strict) linear orders $<_{1}$ and $<_{2}$; so, the permutation on $A=\{a, b, c, k, l\}$ represented by the sequence black would correspond to a pair of chains on $A$ where $a<_{1} b<_{1} c<_{1} k<_{1} l$ (the basic alphabetic order) and $b<_{2} l<_{2}$ $a<_{2} c<_{2} k$. Such an approach proved to be very fruitful in contemporary combinatorics, allowing the study of patterns within permutations [20] and related questions.

To make the notion of homomorphism-homogeneity meaningful in the finite case, here we define a permutation on $A$ to be a structure $\pi=\left(A, \leqslant_{1}, \leqslant_{2}\right)$, where $\leqslant_{1}$ and $\leqslant_{2}$ are two reflexive total orders of $A$; of course, this is conceptually no different from the original definition. However, it will turn useful for our purpose to propose a technically different (yet equivalent) view of a permutation: for a permutation $\pi$ we define a structure

$$
\mathcal{P}_{\pi}=\left(A, \sqsubseteq_{1}, \sqsubseteq_{2}, \leqslant\right)
$$

such that $\leqslant$ is $\leqslant_{1}$ and $\sqsubseteq_{1}, \sqsubseteq_{2}$ are two partial orders on $A$ defined for $a, b \in A$ so that $a \sqsubseteq_{1} b$ if $(a, b)$ does not form an inversion in $\pi$ (that is, if $a \leqslant_{1} b$ and $a \leqslant_{2} b$ ), while otherwise (if $a \leqslant_{1} b$ and $b \leqslant_{2} a$ ) we set $a \sqsubseteq_{2} b$. Conversely, it is fairly routine to see that for any structure of the form $\mathcal{A}=\left(A, \sqsubseteq_{1}, \sqsubseteq_{2}, \leqslant\right)$ such that $\leqslant$ is a linear order of $A$, while $\sqsubseteq_{1}, \sqsubseteq_{2}$ are two partial orders of $A$ such that $\Xi_{1} \cup \Xi_{2}=\leqslant$ and $\Xi_{1} \cap \Xi_{2}$ is the equality relation on $A$, there is a unique permutation $\pi$ on $A$ such that $\mathcal{P}_{\pi}=\mathcal{A}$. This simple idea has its roots in the notion of permutation graphs, tracing back to $[27,17]$. It will turn out that $\pi$ is homomorphism-homogeneous if and only if $\mathcal{P}_{\pi}$ is homomorphism-homogeneous, so that we may completely switch to this latter, alternative approach of colouring the non-loop 
edges of the chain $(A, \leqslant)$ (considered as a directed graph with loops) in two colours, so that each colour induces a partially ordered set on $A$. In this way, the linear order $(A, \leqslant)$ 'splits' into two posets $\left(A, \sqsubseteq_{1}\right)$ and $\left(A, \sqsubseteq_{2}\right)$, to be called blue and red, respectively, and this splitting captures a permutation on $A$ in a unique sense. These edge colourings of chains can be depicted in such a way that the linear order between the points is determined by their vertical positions in the diagram, while the Hasse diagrams of $\left(A, \sqsubseteq_{1}\right)$ and $\left(A, \sqsubseteq_{2}\right)$ are 'superimposed'; full lines will represent the blue order relation, the dotted lines being reserved for the red one. For example, the diagram corresponding to the permutation black mentioned previously is given in Fig. 1.



Figure 1: Permutation black on $\{a, b, c, k, l\}$ represented as an edge coloured chain

Apart from this introductory section, the present note contains another two sections. In the next one we gather all the remaining definitions and prerequisites needed to formulate and prove our main result, Theorem 3 below, which is given in terms of operations $\oplus$ and $\ominus$ of direct and skew sums, respectively, applied to monotone (identical and dual identical) permutations; this is subsequently replaced by more 'economical' modular decompositions. The proof of this theorem occupies Sec. 3. We close the note by another passage from Auster's The New York Trilogy counterpointing nicely the one opening this introduction, and, in a metaphorical way, summing up the combinatorial interplay of colours in our statements and proofs.

\section{Preliminaries and formulation of the main result}

We start this section by justifying the foreshadowed 'categorical equivalence' between permutations as pairs of (reflexive) linear orders on a set and edge colourings of a single chain in blue and red; our aim is to arrive at the conclusion that a permutation $\pi$ is homomorphism-homogeneous if and only if $\mathcal{P}_{\pi}$ is homomorphism-homogeneous. To this end, it suffices to show that the notions of partial endomorphisms coincide in $\pi$ and $\mathcal{P}_{\pi}$. Recall that a homomorphism $f: \mathcal{A} \rightarrow \mathcal{B}$ of relational structures of the same similarity type $\tau$ is a mapping between their underlying sets preserving their relations in the sense that for each $n$-ary relational symbol $R \in \tau$ we have that $\left(a_{1}, \ldots, a_{n}\right) \in R^{\mathcal{A}}$ implies $\left(f\left(a_{1}\right), \ldots, f\left(a_{n}\right)\right) \in R^{\mathcal{B}}$. A bijective homomorphism $f: \mathcal{A} \rightarrow \mathcal{B}$ such that $f^{-1}$ is also 
a homomorphism is an isomorphism. A homomorphism (isomorphism) of a structure $\mathcal{A}$ into itself is an endomorphism (resp. automorphism). We add the adjective 'partial' to these morphisms whenever the domain of $f$ is restricted to some (induced) substructure of $\mathcal{A}$.

Lemma 1. Let $\pi=\left(A, \leqslant_{1}, \leqslant_{2}\right)$ be a permutation on a non-empty set $A$, and let $B \subseteq A$. The the function $f: B \rightarrow A$ is a partial endomorphism of $\pi$ if and only if it is a partial endomorphism of $\mathcal{P}_{\pi}$.

Proof. Let $f: B \rightarrow A$ be a partial endomorphism of $\pi$. To prove that it is also a partial endomorphism of $\mathcal{P}_{\pi}$, it suffices to check that it preserves both $\bigsqcup_{1}$ and $\bigsqcup_{2}$. For example, assume that $b, b^{\prime} \in B$ are such that $b \sqsubseteq_{1} b^{\prime}$. Then $b \leqslant_{1} b^{\prime}$ and $b \leqslant_{2} b^{\prime}$, so $f(b) \leqslant_{1} f\left(b^{\prime}\right)$ and $f(b) \leqslant_{2} f\left(b^{\prime}\right)$. Hence, $f(b) \sqsubseteq_{1} f\left(b^{\prime}\right)$; the argument is analogous for the red order $\sqsubseteq_{2}$. Conversely, assume that $f: B \rightarrow A$ is a partial endomorphism of $\mathcal{P}_{\pi}$. It is immediate that $f$ preserves $\leqslant_{1}$, the first (basic) linear order of $\pi$. Therefore, assume that $b \leqslant_{2} b^{\prime}$ for some $b, b^{\prime} \in B$. If $b \leqslant_{1} b^{\prime}$, then $b \sqsubseteq_{1} b^{\prime}$, so $f(b) \sqsubseteq_{1} f\left(b^{\prime}\right)$. Since we must have $f(b) \leqslant_{1} f\left(b^{\prime}\right)$, we conclude that $f(b) \leqslant_{2} f\left(b^{\prime}\right)$. If, however, $b^{\prime} \leqslant_{1} b$, then $b^{\prime} \sqsubseteq_{2} b$, whence the fact that $f$ preserves $\sqsubseteq_{2}$ yields the same conclusion $f(b) \leqslant_{2} f\left(b^{\prime}\right)$. Therefore, $f$ is a partial endomorphism of $\pi$.

Proposition 2. A permutation $\pi=\left(A, \leqslant_{1}, \leqslant_{2}\right)$ is (homomorphism-)homogeneous if and only if the structure $\mathcal{P}_{\pi}$ is (homomorphism-)homogeneous.

Proof. Assume $\pi$ is a homomorphism-homogeneous permutation; let $f: B \rightarrow f(B) \subseteq A$ be a partial endomorphism of $\mathcal{P}_{\pi}$. Then, by Lemma $1, f$ is a partial endomorphism of $\pi$ (that is, a homomorphism between its finite substructures), so it extends to an endomorphism $f^{*}$ of $\pi$. Applying Lemma 1 again, we have that $f^{*}$ is an endomorphism of $\mathcal{P}_{\pi}$ extending $f$. The converse argument is similar. Also, it is straightforward to see that one can replace (partial) endomorphisms by (partial) automorphisms in the previous argument.

It is thus justified, for our purpose, to identify $\pi$ and $\mathcal{P}_{\pi}$. However, it turns out that the inversion biposet $\mathcal{P}_{\pi}$ will give us a better 'resolution' for the considered problem.

For a permutation $\pi=\left(A, \leqslant_{1}, \leqslant_{2}\right)$, we denote by $\bar{\pi}$ its dual permutation (also called the complement), obtained by reversing the second linear order: $\bar{\pi}=\left(A, \leqslant_{1}, \geqslant_{2}\right)$. In the alternative approach we have $\mathcal{P}_{\bar{\pi}}=\left(A, \sqsubseteq_{2}, \sqsubseteq_{1}, \leqslant\right)$, which amounts to switching between blue and red. The identical permutation on a $k$-element set is denoted by $\iota_{k}$.

For finite permutations $\pi$ and $\sigma$ of length $p$ and $s$, respectively, we define their direct sum $\pi \oplus \sigma$ and skew sum $\pi \ominus \sigma$ as permutations of length $p+s$ given by

$$
\begin{aligned}
& (\pi \oplus \sigma)(i)= \begin{cases}\pi(i) & \text { for } 1 \leqslant i \leqslant p, \\
\sigma(i-p)+p & \text { for } p+1 \leqslant i \leqslant p+s,\end{cases} \\
& (\pi \ominus \sigma)(i)= \begin{cases}\pi(i)+s & \text { for } 1 \leqslant i \leqslant p, \\
\sigma(i-p) & \text { for } p+1 \leqslant i \leqslant p+s .\end{cases}
\end{aligned}
$$

THE ELECTRONIC JOURNAL OF COMBINATORICS 19(4) (2012), \#P17 
An excellent visual example of applications of these operations is given in [1, Fig. 1].

We are now in position to state the main result of this note.

Theorem 3. Let $\pi$ be a permutation on a finite set of $n \geqslant 1$ elements. Then $\pi$ is homomorphism-homogeneous if and only if either $\pi=\overline{\iota_{r_{1}}} \oplus \cdots \oplus \overline{\iota_{r_{m}}}$, or $\pi=\iota_{r_{1}} \ominus \cdots \ominus \iota_{r_{m}}$, where the sequence $\left(r_{1}, \ldots, r_{m}\right)$ satisfies one of the following conditions:

(i) $m=n$ and $r_{1}=\cdots=r_{n}=1$;

(ii) $m \geqslant 2, r_{1}=\cdots=r_{m-1}=1$ and $r_{m}>1$;

(iii) $m \geqslant 2, r_{1}>1$ and $r_{2}=\cdots=r_{m}=1$;

(iv) $m \geqslant 4$ and there exists an index $j$ such that $2 \leqslant j \leqslant m-2, r_{j}, r_{j+1}>1, r_{1}=\cdots=$ $r_{j-1}=1$ and $r_{j+2}=\cdots=r_{m}=1$;

(v) $m \geqslant 3, r_{1}=r_{m}=1$, and for each $j$ such that $2 \leqslant j \leqslant m-2$ we have $1 \in\left\{r_{j}, r_{j+1}\right\}$.

Of course, such a formulation of our result is influenced by the subsequent proof and the need for some sense of uniformity through these five classes, but some cases may be simplified in an obvious manner. For example, (i) simply describes $\iota_{n}$ and its dual $\overline{\iota_{n}}$. Case (ii) comprises the permutations of the form $\iota_{m-1} \oplus \overline{\iota_{r_{m}}}$ and $\overline{\iota_{m-1}} \ominus \iota_{r_{m}}$; dually, case (iii) describes the permutations of the form $\overline{\iota_{r_{1}}} \oplus \iota_{m-1}$ and $\iota_{r_{1}} \ominus \overline{\iota_{m-1}}$; case (iv) is about the permutations of the form $\iota_{j-1} \oplus \overline{\iota_{r_{j}}} \oplus \overline{\iota_{r_{j+1}}} \oplus \iota_{m-j-1}$ and $\overline{\iota_{j-1}} \ominus \iota_{r_{j}} \ominus \iota_{r_{j+1}} \ominus \overline{\iota_{m-j-1}}$.

Notice that all of the permutations involved in the previous theorem belong to the second level of hierarchy of separable permutations (studied, e.g., in $[1,2,4]$ ), obtained from the trivial permutation (with a one-element base set) by repeatedly applying the operations of $\oplus$ and $\ominus$.

Before we launch into proving our main result, it is also worth noting the computational complexity of the problem of recognising homomorphism-homogeneous permutations.

Corollary 4. The question whether a given permutation on an n-element set enjoys the property of homomorphism-homogeneity can be decided in linear time.

\section{$3 \quad$ Proof of Theorem 3}

\subsection{Necessity}

If $\mathcal{P}_{\pi}=\left(A, \sqsubseteq_{1}, \sqsubseteq_{2}, \leqslant\right)$ is a permutation then the blue poset $\left(A, \sqsubseteq_{1}\right)$ will be denoted by $\mathrm{B}_{\pi}$ and the red one $\left(A, \sqsubseteq_{2}\right)$ by $\mathrm{R}_{\pi}$. Our first aim is to prove the following auxiliary result, which provides an explanation why is the notion of $\mathcal{P}_{\pi}$ so useful in the present context.

Proposition 5. If $\mathcal{P}_{\pi}$ is a homomorphism-homogeneous permutation, then both $\mathrm{B}_{\pi}$ and $\mathrm{R}_{\pi}$ are homomorphism-homogeneous posets. 
The proof of this proposition bears a strong resemblance to that of the main result of [22], Theorem 4.5, which characterises homomorphism-homogeneous posets of arbitrary cardinality. Therefore, here is the appropriate place to state this result.

Recall (e.g. from [10], which is the reference to which we direct for all basic notions from order theory) that a tree is a connected partially ordered set in which each down-set $\downarrow x$, consisting of all $y$ such that $y \leqslant x$, is a chain; the connectedness condition simply asserts that any pair (and thus any finite set) of elements has a lower bound. A poset $(A, \leqslant)$ is a dual tree if $(A, \geqslant)$ is a tree. We say that a poset $(A, \leqslant)$ is locally bounded if every finite $X \subseteq A$ has both a lower and an upper bound. An ideal of $(A, \leqslant)$ is a non-empty subset $I \subseteq A$ with the property that $I$ contains the down-set of each of its elements; a filter of $(A, \leqslant)$ is an ideal of $(A, \geqslant)$. A partially ordered set $(A, \leqslant)$ splits into a tree and a dual tree if $A$ can be partitioned into an ideal $I$ that is a tree and a filter $F$ that is a dual tree, such that for each $x \in I$ there is an element $y \in F$ satisfying $x<y$, and, conversely, for each $y \in F$ there is an $x \in I$ with $y>x$. As proved in [22, Lemma 2.4], this is equivalent to the condition that $(A, \leqslant)$ is locally bounded and omits the following substructure, denoted by $\mathcal{X}_{5}$ :

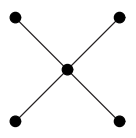

Finally, $(A, \leqslant)$ is said to be $\mathcal{X}_{5}$-dense if for any $a_{1}, a_{2}, b_{1}, b_{2} \in A$ such that $a_{i} \leqslant b_{j}$ for any $i, j \in\{1,2\}$ there exists an element $c \in A$ such that $a_{i} \leqslant c \leqslant b_{j}$ for all $i, j \in\{1,2\}$.

Theorem 6 (Mašulović [22]). A partially ordered set $(A, \leqslant)$ is homomorphism-homogeneous if and only if one of the following conditions holds:

(1) each connected component of $(A, \leqslant)$ is a chain;

(2) $(A, \leqslant)$ is a tree;

(3) $(A, \leqslant)$ is a dual tree;

(4) $(A, \leqslant)$ splits into a tree and a dual tree;

(5) $(A, \leqslant)$ is locally bounded and $\mathcal{X}_{5}$-dense.

As remarked in [22], if $A$ is finite, then (5) is equivalent to the condition that $(A, \leqslant)$ is a lattice.

Proof of Proposition 5. To facilitate the discussion, we first introduce three permutations $\Lambda_{3}, V_{3}$ and $X_{5}$ represented as edge coloured linear orders, whose diagrams are given in Fig. 2. We proceed by distinguishing between various cases depending on whether $\mathcal{P}_{\pi}=\left(A, \sqsubseteq_{1}, \sqsubseteq_{2}, \leqslant\right)$ contains substructures isomorphic to some of the depicted ones. Our aim is to prove that, for example, the blue poset $B_{\pi}$ must be homomorphism-homogeneous, while the proof for $\mathrm{R}_{\pi}$ is analogous. 

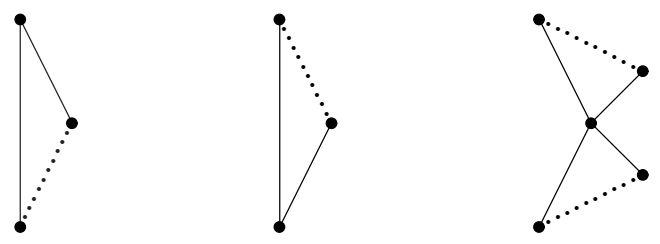

Figure 2: Permutations 213, 132 and 21354 represented as edge-coloured chains: $\Lambda_{3}, V_{3}$ and $X_{5}$

First of all, if $\mathcal{P}_{\pi}$ does not contain a copy of any of $\Lambda_{3}$ or $V_{3}$, then it is immediate that each connected component $B_{\pi}$ must be a chain, so we have case (1) from Theorem 6 .

Now assume that $\mathcal{P}_{\pi}$ contains a copy of $V_{3}$, while it omits $\Lambda_{3}$. We need the analogue of Lemma 4.1 from [22], with a slight modification in the proof.

Claim. If the structure $\mathcal{P}_{\pi}=\left(A, \sqsubseteq_{1}, \sqsubseteq_{2}, \leqslant\right)$ is homomorphism-homogeneous and contains a copy of $V_{3}$, then each finite subset of $A$ has a blue lower bound (that is, a lower bound in $\mathrm{B}_{\pi}$ ).

Proof (Claim). Let $B \subseteq A$ be finite. Consider the blue partial order restricted to the subset $B$ : this induces a partially ordered set $\left(B, \sqsubseteq_{1}\right)$. Let $M$ be the set of all minimal elements of the latter poset. Notice that since no two different $m, m^{\prime} \in M$ are comparable, the elements of $M$ form a red chain in $\mathcal{P}_{\pi}$. Now we prove the claim by induction for the particular case when the finite subset $M$ in question forms a red chain. Indeed, this is trivial if $|M|=1$. If $|M|=2, M=\left\{m_{1}, m_{2}\right\}$ and $m_{1}<m_{2}$, then the induced substructure of $\mathcal{P}_{\pi}$ on $M$ is actually isomorphic to the one induced by the upper two elements $v<w$ of $V_{3}$ (a copy of which is contained somewhere in $\mathcal{P}_{\pi}$ ), so the mapping $f: v \mapsto m_{1}, w \mapsto m_{2}$ is a partial endomorphism. By the given condition, it extends to a total endomorphism $f^{*}$ of $\mathcal{P}_{\pi}$, so if $u$ is the bottom element of $V_{3}$, then $f^{*}(u) \sqsubseteq_{1} f^{*}(v)=f(v)=m_{1}$ and $f^{*}(u) \sqsubseteq_{1} f^{*}(w)=f(w)=m_{2}$. Hence $f^{*}(u)$ is a blue lower bound for $M$ in $\mathcal{P}_{\pi}$.

In the general case, when $|M|>2$, we proceed by induction. Pick $m \in M$ arbitrarily and let $M^{\prime}=M \backslash\{m\}$. Now $M^{\prime}$ is a red chain in $\mathcal{P}_{\pi}$ and $\left|M^{\prime}\right|<|M|$, so by the induction hypothesis $M^{\prime}$ has a blue lower bound $b^{\prime} \in A$. If $b^{\prime} \sqsubseteq_{1} m$, then $b^{\prime}$ is a blue lower bound for the entire $M$; otherwise (since $m \sqsubseteq_{1} b^{\prime}$ would contradict the assumption that $M$ is a red chain), the edge formed by $b^{\prime}$ and $m$ must be red. However, then we already know that the set $\left\{b^{\prime}, m\right\}$ has a blue lower bound $b$ in $\mathcal{P}_{\pi}$. This is clearly a blue lower bound for $M$, thus the inductive argument is complete.

As $\mathcal{P}_{\pi}$ does not contain a copy of $\Lambda_{3}$, it follows that each down-set in $B_{\pi}$ ('blue downset') is a chain. Together with the previous claim, we obtain that $B_{\pi}$ is a tree. Dually, if $\mathcal{P}_{\pi}$ contains a copy of $\Lambda_{3}$, but not of $V_{3}$, it follows that the blue poset $\mathrm{B}_{\pi}$ is a dual tree.

For the remainder of the proof, assume that $\mathcal{P}_{\pi}$ contains copies of both $\Lambda_{3}$ and $V_{3}$. By the previous claim and its dual statement, $\mathcal{P}_{\pi}$ is locally bounded. If $\mathcal{P}_{\pi}$ omits $X_{5}$, then $\mathrm{B}_{\pi}$ omits $\mathcal{X}_{5}$, because there is only one way (up to isomorphism) to 'enrich' the poset $\mathcal{X}_{5}$ to a permutation represented as an edge coloured chain: the two top elements of $\mathcal{X}_{5}$ must 
form a red chain of length 2 , as well as the two bottom elements. Thus, by [22, Lemma 2.4], $\mathrm{B}_{\pi}$ splits into a tree and a dual tree. Finally, if $\mathcal{P}_{\pi}$ contains a copy of $X_{5}$, then the proof of [22, Lemma 4.4] applies verbatim to show that the poset $B_{\pi}$ is $\mathcal{X}_{5}$-dense.

The following remark that applies to the finite case constitutes a key step in simplifying further classification.

Lemma 7. If a finite permutation $\mathcal{P}_{\pi}=\left(A, \sqsubseteq_{1}, \sqsubseteq_{2}, \leqslant\right)$ is homomorphism-homogeneous and $|A| \geqslant 2$, then at least one of the posets $\mathrm{B}_{\pi}$ and $\mathrm{R}_{\pi}$ must be disconnected and thus a free sum of (at least two) chains.

Proof. If $\mathrm{B}_{\pi}$ is disconnected, with each of the connected components being a chain, then there is nothing to prove. On the other hand, if $B_{\pi}$ is a chain, then $R_{\pi}$ must be an antichain with at least two elements, and thus a free sum of singleton chains. Otherwise, $\mathrm{B}_{\pi}$ satisfies one of the conditions (2)-(5) of Theorem 6 , and in each of these cases, $\mathrm{B}_{\pi}$ has either a least, or a greatest element $x$ (this applies to (5) as well, since $A$ is finite, see the remark following Theorem 6). Hence, $x$ is necessarily disconnected from $A \backslash\{x\}$ in $\mathrm{R}_{\pi}$; as the latter must be a homomorphism-homogeneous poset by Proposition 5, each of its components is a chain (by (1) of Theorem 6).

By duality, it will present no loss of generality to assume in the subsequent discussion that the blue poset $\mathrm{B}_{\pi}$ of a finite homomorphism-homogeneous permutation satisfies the condition (1) from Theorem 6, while we analyse what additional conditions can be inferred from the red poset $\mathrm{R}_{\pi}$ being of one of the forms (1)-(5). Such a setup immediately implies that we are dealing with a permutation of the form $\pi=\iota_{r_{1}} \ominus \cdots \ominus \iota_{r_{m}}$ for some sequence of positive integers $\left(r_{1}, \ldots, r_{m}\right)$ such that $r_{1}+\cdots+r_{m}=n=|\pi|$ : if $A=C_{1} \cup \cdots \cup C_{m}$ is the partition of $A$ into maximal blue chains and $r_{i}=\left|C_{i}\right|$ for all $1 \leqslant i \leqslant m$, then a blue chain corresponds to an identical permutation, and the fact that $a, a^{\prime}$ are connected by a red non-loop edge (i.e. that they form an inversion) if and only if $a \in C_{i}$ and $a^{\prime} \in C_{j}$ such that $i \neq j$ means that the identical permutations corresponding to $C_{i}$ 's are combined by the operation $\ominus$ of the skew sum. Furthermore, we fix the convention that if $a \in C_{i}$ and $b \in C_{j}$, then $a \sqsubseteq_{2} b$ if and only if $i<j$. It will turn out that the cases (1)-(5) of Theorem 6 holding for $\mathrm{R}_{\pi}$ will transform into cases (i)-(v) of our Theorem 3.

Case 1: $\mathrm{R}_{\pi}$ is a free sum of chains. If $\mathrm{B}_{\pi}$ is an anti-chain (a free sum of singleton chains), then $\mathrm{R}_{\pi}$ is a chain, thus $\pi=\overline{\iota_{n}}$. Otherwise, $\mathrm{B}_{\pi}$ contains a non-singleton component: say, $a, a^{\prime} \in C_{i}, a \neq a^{\prime}$. If there were another component $C_{j}$ in $\mathrm{B}_{\pi}$ and $b \in C_{j}$, then both edges $(a, b)$ and $\left(a^{\prime}, b\right)$ would be red, while $a$ and $a^{\prime}$ would be incomparable in $\mathrm{R}_{\pi}$, contradicting the assumption on the form of $R_{\pi}$. Hence, $B_{\pi}$ is a single chain and $R_{\pi}$ is an anti-chain, i.e. $\pi=\iota_{n}$. In other words, we have case (i).

Case 2: $\mathrm{R}_{\pi}$ is a tree. If $C_{i}$ fails to be a singleton for some $i \leqslant m-1$, then the red down-set of any $a \in C_{i+1}$ fails to be a chain, contradicting our assumption. Hence, $r_{1}=\cdots=r_{m-1}$. The subcase when $r_{m}=1$ as well is covered by (i), while if $r_{m}>1$ we have precisely case (ii). 
Case 3: $\mathrm{R}_{\pi}$ is a dual tree. Analogously as in the previous paragraph, this situation corresponds to the case (iii) of Theorem 3.

Case 4: $\mathrm{R}_{\pi}$ splits into a tree and a dual tree. In this case, there is an index $j$, $1 \leqslant j<m$, such that the red poset induced on the set $C_{1} \cup \cdots \cup C_{j}$ is a tree, while the red poset induced on $C_{j+1} \cup \cdots \cup C_{m}$ is a dual tree. Now similarly as in the previous two cases, the only non-singleton classes (blue chains) are found among $C_{j}, C_{j+1}$. The possibility when $r_{j}=r_{j+1}=1$ is covered by the first case, while if exactly one of $C_{j}, C_{j+1}$ is nonsingleton, then $\mathrm{R}_{\pi}$ is a lattice, and this belongs to the next case. Finally, if $r_{j}, r_{j+1}>1$, then we must have $2 \leqslant j \leqslant m-2$ (because $r_{1}=r_{m}=1$ by the connectedness requirement of the tree and dual tree substructures of $\mathrm{R}_{\pi}$ ), thus arriving at case (iv).

Case 5: $\mathrm{R}_{\pi}$ is a lattice. Since a finite lattice necessarily has a top and a bottom element, we must have $r_{1}=r_{m}=1$. Now assume that $r_{j}>1$ for some $2 \leqslant j \leqslant m-1$. Since any element of $C_{j+1}$ is an upper bound for any subset of $C_{j}$, and any two elements of $\mathrm{R}_{\pi}$ must have a supremum (a least upper bound), it follows that $r_{j+1}=1$. Analogously, we have $r_{j-1}=1$. Therefore, the situation when $r_{k}, r_{k+1}>1$ for some $k$ (i.e. when two blue chains bearing adjacent indices are both non-singleton) is impossible, which means that we have case $(\mathrm{v})$.

The proof of the implication $(\Rightarrow)$ in Theorem 3 is now complete.

\subsection{Sufficiency}

The implication $(\Leftarrow)$ of Theorem 3 consists in verifying that each of the permutations described in (i)-(v) is homomorphism-homogeneous. To achieve this, we stick with their 'two-colour representation' $\mathcal{P}_{\pi}$ as listed in cases $1-5$ at the end of the previous subsection. The required verification will be performed in the course of the following lemmata. Again, as it is obvious from the representation $\mathcal{P}_{\pi}$ that a permutation $\pi$ is homomorphismhomogeneous if and only if the same holds for its dual $\bar{\pi}$, we will restrict our attention only to permutations of the form $\pi=\iota_{r_{1}} \ominus \cdots \ominus \iota_{r_{m}}$, where the sequence $\left(r_{1}, \ldots, r_{m}\right)$ satisfies one of the conditions from Theorem 3 .

Lemma 8. The identical permutation $\iota_{n}$ is homomorphism-homogeneous.

Proof. Since $\mathrm{R}_{\iota_{n}}$ is just an anti-chain on an $n$-element set $A$, where any self-map of that set is a endomorphism, the question of homomorphism-homogeneity of $\iota_{n}$ reduces to the same question for the chain $\mathrm{B}_{\pi}=\left(A, \sqsubseteq_{1}\right)=(A, \leqslant)$. However, the latter is an immediate consequence of $[22$, Theorem 2.1].

Lemma 9. A permutation of the form $\pi=\iota_{1} \ominus \cdots \ominus \iota_{1} \ominus \iota_{r}=\overline{\iota_{q}} \ominus \iota_{r}, q \geqslant 1, r>1$, is homomorphism-homogeneous.

Proof. With the notation as above, the blue poset $\mathrm{B}_{\pi}$ of $\pi$ (defined on the set $A=$ $\{1, \ldots, q+r\})$ consists of singleton chains $C_{1}, \ldots, C_{q}$ and $C_{q+1}=B$ such that $\left|C_{q+1}\right|=r$; then $R=C_{1} \cup \cdots \cup C_{q}$ is a red chain. Let $f$ be a partial endomorphism of $\pi$ whose domain is $D \subseteq A$. 
Now we describe an extension $f^{*}$ of $f$ to $A$. Let $\downarrow a$ and $\uparrow a$ denote the down-set and the up-set, respectively, of $a \in A$ with respect to the total order $\leqslant$ of $\mathcal{P}_{\pi}$. If $x \in R$ define

$$
f^{*}(x)= \begin{cases}f(\max \downarrow x \cap D) & \text { if } \downarrow x \cap D \neq \varnothing, \\ 1 & \text { otherwise, }\end{cases}
$$

while for $x \in B$ we set

$$
f^{*}(x)= \begin{cases}f(\min \uparrow x \cap D) & \text { if } \uparrow x \cap D \neq \varnothing, \\ f(\max \downarrow x \cap D) & \text { otherwise. }\end{cases}
$$

We need to prove that $f^{*}$ preserves both the blue partial order $\sqsubseteq_{1}$ and the red order $\sqsubseteq_{2}$. Indeed, assume that $x \sqsubseteq_{1} y$ and $x \neq y$. Then, since $B$ is the only non-singleton component of $\mathrm{B}_{\pi}$, we have $x, y \in B$. If $\uparrow y \cap D=\varnothing$, then $f^{*}(y)=f(\max \downarrow y \cap D)$. If the interval $] x, y\left[=\{z: x<z<y\}\right.$ contains no element of $D$, then clearly $f^{*}(x)=f^{*}(y)$; otherwise, $\max \downarrow y \cap D \geqslant \min \uparrow x \cap D$, and since $f$ is a partial endomorphism of $\mathcal{P}_{\pi}$, we have

$$
f^{*}(x)=f(\min \uparrow x \cap D) \leqslant f(\max \downarrow y \cap D)=f^{*}(y) .
$$

However, both min $\uparrow x \cap D$ and $\max \downarrow y \cap D$ belong to $] x, y[$ and thus to $B$, so the edge determined by them is either a loop or blue. Therefore, the $f$-image of that edge, which is $\left(f^{*}(x), f^{*}(y)\right)$, must be also a loop or blue, i.e. $f^{*}(x) \sqsubseteq_{1} f^{*}(y)$. On the other hand, if $\uparrow y$ contains an element of $D$, then the same holds for $\uparrow x$, and since $\min \uparrow x \cap D \leqslant \min \uparrow y \cap D$, we obtain

$$
f^{*}(x)=f(\min \uparrow x \cap D) \leqslant f(\min \uparrow y \cap D)=f^{*}(y) .
$$

Again, since min $\uparrow x \cap D, \min \uparrow y \cap D \in B$, we conclude once more that $f^{*}(x) \sqsubseteq_{1} f^{*}(y)$.

Now let $x, y \in A$ be such that $x \sqsubseteq_{2} y$ and $x \neq y$; then $x \in R$. If $\downarrow x \cap D=\varnothing$, then $f^{*}(x)=1$, which instantly yields $f^{*}(x) \sqsubseteq_{2} f^{*}(y)$ (as 1 is the least element of $A$ and is connected by a red edge to any other element, since $\left.C_{1}=\{1\}\right)$. Hence, we may assume that $\downarrow x \cap D \neq \varnothing$, implying $f^{*}(x)=f(\max \downarrow x \cap D)$ and $\downarrow y \cap D \neq \varnothing$. So, if either $y \in R$, or $y \in B$ and $\uparrow y \cap D=\varnothing$, then $f^{*}(y)=f(\max \downarrow y \cap D)$; otherwise (if $y \in B$ and $\uparrow y \cap D \neq \varnothing)$ we have $f^{*}(y)=f(\min \uparrow y \cap D)$. But in any case we must have $\max \downarrow x \cap D \in R$, as $x \in R$, thus max $\downarrow x \cap D$ is necessarily connected by a loop or a red edge both to $\max \downarrow y \cap D \geqslant \max \downarrow x \cap D$ and to $\min \uparrow y \cap D \geqslant y \geqslant x \geqslant \max \downarrow x \cap D$. This immediately implies $f^{*}(x) \sqsubseteq_{2} f^{*}(y)$, so we are done.

The following lemma is proved in a completely analogous manner, and thus its verification is left to the reader.

Lemma 10. A permutation of the form $\pi=\iota_{r} \ominus \iota_{1} \cdots \ominus \iota_{1}=\iota_{r} \ominus \overline{\iota_{q}}, q \geqslant 1, r>1$, is homomorphism-homogeneous.

Lemma 11. A permutation of the form $\pi=\overline{\iota_{p}} \ominus \iota_{q} \ominus \iota_{r} \ominus \overline{\iota_{s}}, p, s \geqslant 1, q, r>1$, is homomorphism-homogeneous. 
Proof. In a way, the proof of this lemma is a 'synthesis' of the previous two. Here the base set of the permutation $\pi$ is $A=\{1, \ldots, p+q+r+s\}$ (we let $n=p+q+r+s$ ), and $\mathrm{B}_{\pi}$ is a union of (blue) chains $C_{1}=\{1\}, \ldots, C_{s}, C_{s+1}=B^{\prime}, C_{s+2}=B^{\prime \prime}, C_{s+3}, \ldots, C_{s+p+2}=\{n\}$, such that $\left|B^{\prime}\right|=r,\left|B^{\prime \prime}\right|=q$, while all the others are singletons. Furthermore, in $\mathrm{R}_{\pi}$ we have $x \sqsubseteq_{2} y$ if and only if either $x=y$, or $x \in C_{i}, y \in C_{j}$ such that $i<j$. Denote $R^{\prime}=C_{1} \cup \cdots \cup C_{s}, R^{\prime \prime}=C_{s+3} \cup \cdots \cup C_{s+p+2}$ (these form red chains), and assume $f$ is a partial endomorphism of $\mathcal{P}_{\pi}$ with domain $D$. Notice that the restriction of the red order to $R^{\prime} \cup B^{\prime}$ forms a tree which is an ideal of $\mathrm{R}_{\pi}$, while $B^{\prime \prime} \cup R^{\prime \prime}$ is a filter of $\mathrm{R}_{\pi}$ that is a dual tree.

We define an extension $f^{*}$ of $f$ separately on four 'pieces' $R^{\prime}, B^{\prime}, B^{\prime \prime}, R^{\prime \prime}$. Namely, if $x \in R^{\prime}$, then

$$
f^{*}(x)= \begin{cases}f(\max \downarrow x \cap D) & \text { if } \downarrow x \cap D \neq \varnothing \\ 1 & \text { otherwise, }\end{cases}
$$

for $x \in B^{\prime}$ we set

$$
f^{*}(x)= \begin{cases}f\left(\min \uparrow x \cap D \cap B^{\prime}\right) & \text { if } \uparrow x \cap D \cap B^{\prime} \neq \varnothing, \\ f(\max \downarrow x \cap D) & \text { if } \uparrow x \cap D \cap B^{\prime}=\varnothing, \text { but } \downarrow x \cap D \neq \varnothing, \\ 1 & \text { otherwise, }\end{cases}
$$

for $x \in B^{\prime \prime}$ we define

$$
f^{*}(x)= \begin{cases}f\left(\max \downarrow x \cap D \cap B^{\prime \prime}\right) & \text { if } \downarrow x \cap D \cap B^{\prime \prime} \neq \varnothing, \\ f(\min \uparrow x \cap D) & \text { if } \downarrow x \cap D \cap B^{\prime \prime}=\varnothing, \text { but } \uparrow x \cap D \neq \varnothing, \\ n & \text { otherwise, }\end{cases}
$$

and, finally, for $x \in R^{\prime \prime}$ we have

$$
f^{*}(x)= \begin{cases}f(\min \uparrow x \cap D) & \text { if } \uparrow x \cap D \neq \varnothing, \\ n & \text { otherwise. }\end{cases}
$$

The proof that the map $f^{*}$ just constructed preserves both the blue and the red partial order is now fairly similar to the corresponding verification performed in the proof or Lemma 9. Indeed, if $x, y \in A$ are such that $x \sqsubseteq_{1} y$ and $x<y$, then either $x, y \in B^{\prime}$, or $x, y \in B^{\prime \prime}$. A straightforward case analysis would show that whenever $x, y \in B^{\prime}$ and $f^{*}(x) \neq f^{*}(y)$ then there exist elements $d_{1}, d_{2} \in D \cap B^{\prime}$, more precisely $d_{1} \in\{$ min $\uparrow$ $\left.x \cap D \cap B^{\prime}, \max \downarrow x \cap D \cap B^{\prime}\right\}$ and $d_{2} \in\left\{\min \uparrow y \cap D \cap B^{\prime}, \max \downarrow y \cap D \cap B^{\prime}\right\}$, such that $d_{1} \sqsubseteq_{1} d_{2}$ and $f^{*}(x)=f\left(d_{1}\right), f^{*}(y)=f\left(d_{2}\right)$. A similar conclusion follows if $x, y \in B^{\prime \prime}$. By the assumptions placed on $f$, this yields $f^{*}(x) \sqsubseteq_{1} f^{*}(y)$.

On the other hand, let $x<y$ be such that $x \sqsubseteq_{2} y$ (i.e. the edge $(x, y)$ is red). Then $x \in$ $C_{i}, y \in C_{j}$ such that $i<j$. We may assume that $f^{*}(x) \neq 1$ and $f^{*}(y) \neq n$, for otherwise we immediately reach the desired conclusion $x \sqsubseteq_{2} y$. If $x \in R^{\prime} \cup B^{\prime}$ and $y \in B^{\prime \prime} \cup R^{\prime \prime}$, then, by the definition of $f^{*}, f^{*}(x)=f\left(d^{\prime}\right)$ and $f^{*}(y)=f\left(d^{\prime \prime}\right)$ for some $d^{\prime} \in D \cap\left(R^{\prime} \cup B^{\prime}\right)$ and $d^{\prime \prime} \in D \cap\left(B^{\prime \prime} \cup R^{\prime \prime}\right)$, so $d^{\prime} \sqsubseteq_{2} d^{\prime \prime}$ and the fact that $f$ is a partial endomorphism implies 
$f^{*}(x) \sqsubseteq_{2} f^{*}(y)$. Otherwise, either $x, y \in R^{\prime} \cup B^{\prime}$, or $x, y \in R^{\prime \prime} \cup B^{\prime \prime}$; we consider only the former case, since the latter is analogous. By all the assumptions made previously, $f^{*}(x)=f(\max \downarrow x \cap D)$ and $f^{*}(y) \in\left\{f\left(\min \uparrow y \cap D \cap B^{\prime}\right), f(\max \downarrow y \cap D)\right\}$. Since $x<y$, the edge $\left(f^{*}(x), f^{*}(y)\right)$ is the $f$-image of a red edge $(d, \bar{d})$ formed by elements $d<\bar{d}$ of $D$. Therefore, $f^{*}(x) \sqsubseteq_{2} f^{*}(y)$.

We are ready to tackle the most complex case in this subsection, the one determined by condition (v) of Theorem 3 .

Lemma 12. Let $\pi=\iota_{1} \ominus \iota_{p_{1}} \ominus \cdots \ominus \iota_{p_{k}} \ominus \iota_{1}$ such that $1 \in\left\{p_{u}, p_{u+1}\right\}$ for all $1 \leqslant u<k$. Then $\pi$ is homomorphism-homogeneous.

Proof. We start by recording the structure of $\mathrm{B}_{\pi}$ and $\mathrm{R}_{\pi}$ : in the blue poset, we have chains $C_{0}=\{1\}, C_{1}, \ldots, C_{k}, C_{k+1}=\{n\}$ such that $\left|C_{i}\right|=p_{i}$ for $1 \leqslant i \leqslant k$ and $n=p_{1}+\cdots+p_{k}+2$. In the red poset, for $x<y$ we have $x \sqsubseteq_{2} y$ if and only if $x \in C_{i}, y \in C_{j}$ for some $0 \leqslant i<j \leqslant k+1$.

Let us pause to make two important observations on partial endomorphisms of permutations of the considered form; this will aid us significantly in our construction.

Claim 1. Let $f$ be a (partial) endomorphism of $\pi$ (that is, of $\mathcal{P}_{\pi}$ ), while $f^{*}$ is an arbitrary extension of $f$ to a total endomorphism. Then for any $i, 0 \leqslant i \leqslant k+1$, if for some $a \in C_{i}$ we have $f(a) \in C_{j}$ for some $j$, then $f^{*}(x) \in C_{j}$ holds for all $x \in C_{i}$.

Proof (Claim 1). If $x \in C_{i}$, then either $a \sqsubseteq_{1} x$, or $x \sqsubseteq_{1} a$; for the sake of an example, assume that the latter takes place. Then we must have $f^{*}(x) \sqsubseteq_{1} f^{*}(a)=f(a)$; in other words, the edge determined by $f^{*}(x)$ and $f(a) \in C_{j}$ is blue. This is possible only if $f^{*}(x) \in C_{j}$ as well.

The previous claim says that any partial endomorphism $f$ of $\mathcal{P}_{\pi}$ consistently and uniquely defines a partial transformation $\mathbf{t}_{f}$ of the set $\{0,1, \ldots, k+1\}$ that preserves the chain $0 \leqslant 1 \leqslant \ldots \leqslant k+1$, and for any total endomorphism $f^{*}$ extending $f$, $\mathbf{t}_{f^{*}}$ is an order-preserving transformation of that chain. Hence, in the sequel, our strategy in the course of extending a partial endomorphism $f$ will be to first record $\mathbf{t}_{f}$, extend it to a total transformation $\mathbf{t}$ of $\{0,1, \ldots, k+1\}$ in a specific way, and then to use it as a 'guide' in constructing $f^{*}$.

Claim 2. Let $f$ be a partial endomorphism of $\pi$. If $0 \leqslant i \neq i^{\prime} \leqslant k+1$ are such that $\mathbf{t}_{f}(i)=\mathbf{t}_{f}\left(i^{\prime}\right)=j$ for some $j$, then there exists an element $x \in C_{j}$ such that $f(a)=f\left(a^{\prime}\right)=x$ for all $a \in C_{i}, a^{\prime} \in C_{i^{\prime}}$.

Proof (Claim 2). Fix an arbitrary $a_{0} \in C_{i}$ and define $x=f\left(a_{0}\right) \in C_{j}$. Let $a^{\prime} \in C_{i^{\prime}}$ and assume, without loss of generality, that $i<i^{\prime}$. Then $a_{0} \sqsubseteq_{2} a^{\prime}$ (i.e. the edge $\left(a_{0}, a^{\prime}\right)$ is red), so $x=f\left(a_{0}\right) \sqsubseteq_{2} f\left(a^{\prime}\right)$ (the edge $\left(x, f\left(a^{\prime}\right)\right)$ is red as well, or a loop). Since it is postulated that $f\left(a^{\prime}\right) \in C_{j}$, which is the same blue chain that contains $x$, this is possible only if $f\left(a^{\prime}\right)=x$. Similarly, for any $a \in C_{i}$ and $a^{\prime} \in C_{i^{\prime}}$ we now have $a \sqsubseteq_{2} a^{\prime}$, so $C_{j} \ni f(a) \sqsubseteq_{2} f\left(a^{\prime}\right)=x$, which again implies $f(a)=x$. 
Turning back to the main course of the proof, let $f$ be a partial endomorphism of $\pi$ with domain $D$, and let $i_{1}<\cdots<i_{s}$ be a complete list of all indices $i \in\{0, \ldots, k+1\}$ with the property that $D \cap C_{i} \neq \varnothing$. Our first step is to extend $f$ to a partial endomorphism $\bar{f}$ of $\pi$ with domain $\bar{D}=C_{i_{1}} \cup \cdots \cup C_{i_{s}}$. This is done by defining, for $x \in \bar{D}$,

$$
\bar{f}(x)= \begin{cases}f(\max \downarrow x \cap D) & \text { if } \downarrow x \cap D \neq \varnothing, \\ f(\min \uparrow x \cap D) & \text { otherwise. }\end{cases}
$$

Indeed, $\bar{f}$ clearly preserves the blue partial order of $\mathrm{B}_{\pi}$ restricted to $\bar{D}$ (cf. [22, Theorem 2.1]). If, however, $x \in C_{i_{q}}, y \in C_{i_{r}}$ such that $q<r$ (so that $x \sqsubseteq_{2} y$ ), then the edge $(\bar{f}(x), \bar{f}(y))$ is the $f$-image of $\left(x^{\prime}, y^{\prime}\right)$ for some $x^{\prime} \in C_{i_{q}} \cap D, y^{\prime} \in C_{i_{r}} \cap D$, yielding $\bar{f}(x) \sqsubseteq_{2} \bar{f}(y)$.

At this point, we have at our disposal the partial order-preserving transformation $\mathbf{t}_{f}=\mathbf{t}_{\bar{f}}$ of $\{0, \ldots, k+1\}$ whose domain is $I=\left\{i_{1}, \ldots, i_{s}\right\}$. As we announced, the next step is to extend it to a total order-preserving transformation $\mathbf{t}$ in a particular way, bearing in mind Claim 2. Now let $j \notin I$. If $j<i_{1}$ set $\mathbf{t}(j)=0$, while if $j>i_{s}$ put $\mathbf{t}(j)=k+1$. Otherwise, there is a unique index $1 \leqslant t<s$ such that $i_{t}<j<i_{t+1}$. If $\mathbf{t}_{f}\left(i_{t}\right)=\mathbf{t}_{f}\left(i_{t+1}\right)$, then we define $\mathbf{t}(j)=\mathbf{t}_{f}\left(i_{t}\right)$; if, however, $\mathbf{t}_{f}\left(i_{t}\right)<\mathbf{t}_{f}\left(i_{t+1}\right)$, then by the conditions of the lemma, the interval of indices $\left[\mathbf{t}_{f}\left(i_{t}\right), \mathbf{t}_{f}\left(i_{t+1}\right)\right]$ must contain at least one index $\ell$ such that $p_{\ell}=1$, and we let $\mathbf{t}(j)$ to be the least such index.

Now we have set up the stage for extending $\bar{f}$ to $f^{*}$, a total self-map of $A=\{1, \ldots, n\}$. Let $j \notin I$. If $C_{\mathbf{t}(j)}$ is a singleton, then we define $f^{*}(x)$ to be its only element, for any $x \in C_{j}$. Notice that the only possibility for $\left|C_{\mathbf{t}(j)}\right|>1$ occurs when $i_{t}<j<i_{t+1}$ for some $t$ and $\mathbf{t}\left(i_{t}\right)=\mathbf{t}(j)=\mathbf{t}\left(i_{t+1}\right)=w$. Then, however, by Claim 2 (applied to $\bar{f}$ ) we have that $\bar{f}(a)=\bar{f}\left(a^{\prime}\right)=b$ for all $a \in C_{i_{t}}, a^{\prime} \in C_{i_{t+1}}$ and for some $b \in C_{w}$, so let $f^{*}(x)=b$ for all $x \in C_{j}$.

Note that our construction entails that for any $j \notin I, f\left(C_{j}\right)$ is a singleton subset of $C_{\mathbf{t}(j)}$ (in some cases, $C_{\mathbf{t}(j)}$ itself is a singleton set). Therefore, it instantly follows that $f^{*}$ preserves the blue partial order. To prove that the same holds for the red partial order, note first that if either $j_{1}<j_{2}<i_{1}$, or $i_{t}<j_{1}<j_{2}<i_{t+1}$, or $i_{s}<j_{1}<j_{2}$, then $f^{*}(x)=f^{*}(y)$ for any $x \in C_{j_{1}}, y \in C_{j_{2}}$. Hence, by transitivity and the verified fact that $\bar{f}$ is a partial endomorphism, it suffices to look at red edges $x \sqsubseteq_{2} y$ such that either $x \in C_{i_{t}}, y \in C_{j}$, or $x \in C_{j}, y \in C_{i_{t+1}}$, where $i_{t}<j<i_{t+1}$ for some $t$. In the former case, $f^{*}(x)=\bar{f}(x) \in C_{\mathbf{t}\left(i_{t}\right)}$, while $f^{*}(y) \in C_{\mathbf{t}(j)}$. By definition, $f^{*}(x) \neq f^{*}(y)$ implies that $\mathbf{t}\left(i_{t}\right)<\mathbf{t}\left(i_{t+1}\right)$ and that $C_{\mathbf{t}\left(i_{t}\right)}$ is not a singleton. But then $\mathbf{t}\left(i_{t}\right)<\mathbf{t}(j) \leqslant \mathbf{t}\left(i_{t+1}\right)$, whence $f^{*}(x) \sqsubseteq_{2} f^{*}(y)$. The other case is similar, thus the lemma is proved.

This completes the proof of our Theorem 3 , as the implication $(\Leftarrow)$ follows directly from Lemmas 8, 9, 10, 11 and 12 and their dual statements.

On certain nights, when it is clear to Blue that Black will not be going anywhere, he slips out to a bar not far away for a beer or two, enjoying the conversations he 
sometimes has with the bartender, whose name is Red, and who bears an uncanny resemblance to Green, the bartender from the Gray Case so long ago.

Paul Auster: Ghosts (The New York Trilogy)

Acknowledgement. We are grateful to Dragan Mašulović for a careful reading of the first draft of our manuscript. The research of both authors is supported by the Ministry of Education, Science and Technological Development of the Republic of Serbia through Grant No.174019. The first author also acknowledges the support by a grant (Contract 114-451-2675/2012) of the Secretariat of Science and Technological Development of the Autonomous Province of Vojvodina.

\section{References}

[1] M. H. Albert, M. D. Atkinson and V. Vatter, Subclasses of the separable permutations, Bull. London Math. Soc. 43 (2011), 859-870.

[2] D. Avis and M. Newborn, On pop-stacks in series, Util. Math. 19 (1981), 129-140.

[3] D. Bertholf and D. Walls, Finite quasi-injective groups, Glasgow Math. J. 20 (1979), $29-33$.

[4] P. Bose, J. F. Buss and A. Lubiw, Pattern matching for permutations, Inform. Process. Lett. 65 (1998), 277-283.

[5] P. J. Cameron, Homogeneous permutations, Electron. J. Combin. 9 (2002), no.2, \#R2, 9 pp.

[6] P. J. Cameron and D. C. Lockett, Posets, homomorphisms and homogeneity, Discrete Math. 310 (2010), 604-613.

[7] P. J. Cameron and J. Nešetřil, Homomorphism-homogeneous relational structures, Combin. Probab. Comput. 15 (2006), 91-103.

[8] G. Cherlin, The classification of countable homogeneous directed graphs and countable homogeneous n-tournaments, Mem. Amer. Math Soc. 131 (1998), no.621, xiv $+161 \mathrm{pp}$.

[9] G. Cherlin and U. Felgner, Homogeneous finite groups, J. London Math. Soc. (2) 61 (2000), 784-794.

[10] B. A. Davey and H. A. Priestley, Introduction to Lattices and Order, 2nd ed., Cambridge University Press, Cambridge, 2002.

[11] I. Dolinka and D. Mašulović, Remarks on homomorphism-homogeneous lattices and semilattices, Monatsh. Math. 164 (2011), 23-37.

[12] R. Fraïssé, Sur certains relations qui généralisent l'ordre des nombres rationnels, $C$. R. Acad. Sci. Paris 237 (1953), 540-542.

[13] A. D. Gardiner, Homogeneous graphs, J. Combin. Theory Ser. B 20 (1976), 94-102. 
[14] W. Hodges, A Shorter Model Theory, Cambridge University Press, Cambridge, 1997.

[15] A. Ilić, D. Mašulović and U. Rajković, Finite homomorphism-homogeneous tournaments with loops, J. Graph Theory 59 (2008), 45-58.

[16] É. Jungábel and D. Mašulović, Homomorphism-homogeneous monounary algebras, Math. Slovaca (to appear).

[17] Y. Koh and S. Ree, Connected permutation graphs, Discrete Math. 307 (2007), 2628-2635.

[18] A. H. Lachlan, Countable homogeneous tournaments, Trans. Amer. Math. Soc. 284 (1984), 431-461.

[19] A. H. Lachlan and R. E. Woodrow, Countable ultrahomogeneous undirected graphs, Trans. Amer. Math. Soc. 262 (1980), 51-94.

[20] S. A. Linton, N. Ruškuc and V. Vatter (eds.), Permutation Patterns (St Andrews 2007), LMS Lecture Notes Series, Vol. 376, Cambridge University Press, Cambridge, 2010.

[21] D. Macpherson, A survey of homogeneous structures, Discrete Math. 311 (2011), 1599-1634.

[22] D. Mašulović, Homomorphism-homogeneous partially ordered sets, Order 24 (2007), 215-226.

[23] D. Mašulović, Some classes of finite homomorphism-homogeneous point line geometries, Combinatorica (to appear).

[24] D. Mašulović, On the complexity of deciding homomorphism-homogeneity for finite algebras, submitted manuscript, $12 \mathrm{pp}$.

[25] D. Mašulović, R. Nenadov and N. Škorić, On finite reflexive homomorphism-homogeneous binary relational systems, Discrete Math. 311 (2011), 2543-2555.

[26] D. Mašulović and M. Pech, Oligomorphic transformation monoids and homomorphism-homogeneous structures, Fund. Math. 212 (2011), 17-34.

[27] A. Pnueli, A. Lempel and S. Even, Transitive orientation of graphs and identification of permutation graphs, Canad. J. Math. 23 (1971), 160-175.

[28] M. Rusinov and P. Schweitzer, Homomorphism-homogeneous graphs, J. Graph Theory 65 (2010), 253-262.

[29] J. H. Schmerl, Countable homogeneous partially ordered sets, Algebra Universalis 9 (1979), 317-321.

[30] M. J. Tomkinson, Infinite quasi-injective groups, Proc. Edinb. Math. Soc. 31 (1988), 249-259. 